\title{
Cisj
}

\section{IDENTIFICATION AND FORECASTING OF SHARP CHANGES IN ECONOMICAL SYSTEMS BY TRACKING OF LOCAL LYAPUNOV EXPONENTS}

\author{
Dmitry Malyuk ${ }^{1)}$, Georgy Boyarintsev ${ }^{2)}$ \\ 1) Brest State Technical University, 224017, Republic of Belarus, Brest, \\ Moskovskaya st., 267, rodjko.o.v@mail.ru \\ 2) Brest State Technical University, 224017, Republic of Belarus, Brest, \\ Moskovskaya st., 267
}

\begin{abstract}
An approach to detect sharp changes in economical systems was developed in this paper. We will give brief introduction in current methods of prediction of nonlinear and chaotic time-series and give definition of local Lyapunov exponents (LLE). Then author's approach will be described. Also some numerical results and discussions will be given.
\end{abstract}

Keywords: sharp changes, unpredictability, local Lyapunov exponents, Takens' theorem

\section{INTRODUCTION}

The problem of forecasting in economics has wide range of sources in literature, which proposes variety of methods. But there are many difficulties arise, when one want to apply any method to realdata analysis.

There are some issues, which would be taken in account, when one wants to apply any method to real economical data:

1. Any economical process driving by many of deterministic and stochastic forces and strictly deterministic so as strictly probabilistic approach is irrelevant.

2. Underlying dynamics is completely unknown in most cases.

3. Receiving the good data for forecasting is a big problem - sometimes only short noisy timeseries of a few aggregate variables.

4. Economical processes are intrinsically nonstationary, and therefore we must be very careful with interpretation of our results.

Last point is especially important and if we don't take it in account then mistake can occurs. Changes in economical environment occur every day - some of them is very sharp and unpredictable. All forecasts of future based on past history, but if dramatic change occurs in dynamics - our forecast would be incorrect at least. What we can do with this?

Remainder of this paper organized as follows. In Section 2 we give some introduction in prediction based on nonlinear dynamics, definition of local Lyapunov exponents, describe implications of Takens' theorem. In Section 3 author describe his approach, give parameters for identification of sharp changes and some points for their prediction. In Section 4 some numerical results and discussion are given.

\section{PRELIMINARIES}

A phenomenon of chaotic behavior occurs in many fields of experimental science. Chaotic dynamics has nonlinear components and characterized by sensitivity to initial conditions that lead to random-like behavior of time-series [1]. Issue about nature of economical cycles is not clear today yet. It is open problem whether is chaos in economics or all fluctuations can be described by random-walk model (see, for example [2], [3])?

One of the widely used tests for chaos is determination of largest Lyapunov exponents [1].

Lyapunov exponents referred as mean effective rate of divergence (convergence) of nearby trajectories along different directions. Consider discrete (continuous) n-dimensional system:

$$
\mathbf{x}_{k+1}=F\left(\mathbf{x}_{k}\right) \quad(\dot{\mathbf{x}}=F(\mathbf{x}))
$$

Sphere in the phase space evolving in $\mathrm{n}$ dimensional ellipsoid. Lyapunov exponents could be expressed: 


$$
\lambda_{i}=\lim _{\Delta t \rightarrow \infty} \frac{1}{\Delta t} \log \left(\frac{\left\|\varepsilon_{i}^{\Delta t}\right\|}{\left\|\varepsilon_{i}^{0}\right\|}\right)
$$

where $\varepsilon_{i}^{0}$ - projection of initial perturbation on the $\mathrm{i}$-th principal axis of ellipsoid, $\varepsilon_{i}^{\Delta t}$ - projection of perturbation on the $i$-th principal axis of ellipsoid after time $\Delta t$ (which could be discrete or continuous) [4].

Also Lyapunov exponents might be considered as singular values of linear propagator $\mathbf{J}^{\Delta t}\left(x_{k}\right)$ :

$$
\mathbf{J}^{\Delta t}\left(x_{k}\right)=\prod_{n=0}^{\Delta t} \mathbf{J}\left(x_{k+n}\right) \quad\left(\mathbf{J}^{\Delta t}\left(x_{k}\right)=e^{\int_{t+\Delta t}^{t} \mathbf{J}(x(t)) d t}\right)
$$

where J - Jacobian matrix of (1) [5], [6]. First (largest) Lyapunov exponent most important of them because it determinate dynamical properties of system. In particular, positive largest Lyapunov exponent imply chaotic behavior.

There are many algorithms for determination of Lyapunov exponents, but if we deal with real-life data then we must reconstruct unknown dynamics from observations. One of the points to decide this problem is Takens' theorem.

Consider discrete system (1) and time-series of observation:

$$
y_{n}=g\left(\mathbf{x}_{n}\right)+w_{n}
$$

where $g(\cdot)$ - scalar-valued measurement function, $w_{n}$ - additive noise.

Consider time-delayed reconstruction of (4):

$$
\mathbf{y}_{k}^{R}=\left[y_{n}, y_{n-\tau}, \ldots, y_{n-(D-1) \tau}\right]^{T}
$$

where $\tau$ - time lag, $D$ - embedding dimension.

It was proved that in condition that time-series of (4) noise-free and has infinite length than with $D \geq 2 d+1$, where $d$ is fractal dimension of original attractor, reconstruction (5) of system has the same geometrical structure that of original system with any $\tau[7]$.

But real data has finite length and precision, and therefore value of lag is very important too.

There are many methods for choosing $\tau$ and $D$. We will be use for determination $\tau$ the first minimum of mutual information, which is:

$$
I(\tau)=\sum_{k=1}^{m-1} P\left(y_{k}, y_{k+\tau}\right) \ln \frac{P\left(y_{k}, y_{k+\tau}\right)}{P\left(y_{k}\right) P\left(y_{k+\tau}\right)}
$$

where $P\left(y_{k}, y_{k+\tau}\right)$ - conditional probability of value $y_{k+\tau}$ at time $k+\tau$ in condition of value $y_{k}$ at time $k, P\left(y_{k}\right), P\left(y_{k+\tau}\right)$ - corresponding marginal probabilities [8].

For determination of embedding dimension we will be use false nearest neighbours method, which are consist of following steps [9].

1.) For every embedding dimension from 2 to some upper limit one build reconstructed time-series.

2.) For every point of reconstructed series one find nearest another point, which are called nearest neighbour.

3.) It is necessary to find

$$
R_{i}=\frac{\left\|y_{i+1}^{R}-y_{j+1}^{R}\right\|}{\left\|y_{i}^{R}-y_{j}^{R}\right\|}
$$

where $y_{j}^{R}$ - the nearest neighbour for $y_{i}^{R}$.

4.) If $R_{i}>R_{c}$ than this pair of points is false nearest neighbours, where $R_{c}$ - some constant.

5.) We may choose embedding dimension for which rate of false nearest neighbours less than some $\varepsilon$.

Author consider $R_{c}=1.5$ and $\varepsilon=0.02$.

Nowadays, there two main approaches for estimating Lyapunov exponents from observed data. First approach based on estimation divergence rate through principal axis of expansion [10] by formula (2), and second - on approximation of tangent map [5] and estimation singular values of (3). One could apply each of them after approximating of underlying dynamics by any model and neural nets one of most popular methods, and it was applied by many authors for estimating of Lyapunov exponents within either first approach [11] or second [12].

Also, it is necessary to stress, that Lyapunov exponents in (2) and (3) has an infinite limits. These exponents are the same for every point and invariant, but it is impossible to calculate infinite limit indeed. Therefore, notion of local Lyapunov exponents appear. The n-step ahead local Lyapunov exponent is

$$
\lambda_{i}^{\Delta t}=\frac{1}{\Delta t} \log \left(\frac{\left\|\varepsilon_{i}^{\Delta t}\right\|}{\left\|\varepsilon_{i}^{0}\right\|}\right)
$$

That is, we have finite number $\Delta t$ instead of infinite limit. Local Lyapunov exponents are not invariant, but they can be applied as a measure of heterogeneity of system [5], [6], [12]. 
In this paper, author attempt to apply local Lyapunov exponents for identification of sharp changes.

\section{IDENTIFICATION OF SHARP CHANGES}

Sharp sudden changes used to occur in economics sufficiently frequently. Saying by words of dynamics, $F$ in system (1) change in some way, and dynamical properties of (1) change too. On the other hand, from practical point of view, these changes are notable, if we can observe sufficient increasing or decreasing of some rate. So we can outline some points about sharp changes:

1.) They caused by some switch in underlying dynamics, which, in turn, caused by some fluctuations in the environment.

2.) They manifest themselves in observed data by variations of means, variance, mean Fourier frequency etc.

3.) Sharpness implies unpredictability.

Third point is very important for forecasting. All forecasts imply delivering of information about future from past data. And if underlying dynamics change drastically, then our forecast fate on failure. Therefore, it is very important detect point of these changes, in order to avoid the fault in forecasts.

For identification such changes author propose using of neural networks to detect periods of unpredictability in time-series. For solution of this problem following algorithm was applied:

1.) Width of moving window $b$ fixed.

2.) Reconstructed vectors (5) created for every piece of time-series of length $b$ in accordance with above-mentioned procedures for finding of $\tau$ and $D$.

3.) Multi-layer network with $D$ input neurons, sufficient number of hidden neurons and single output trained for prediction of next value of series for every piece.

4.) Network simulated by one step-prediction $n$ times and mean absolute error $E(i)$ of prediction estimated, where $i$ - number of piece.

5.) Looking to sufficiently big value of $E(i)$, one could identify point of unpredictability $i+b$. (See Fig. 1).

The natural way to predict manifestation of sharp changes such as jumps, sudden up-and-downs is the early detection of system changes. The objective of this paper

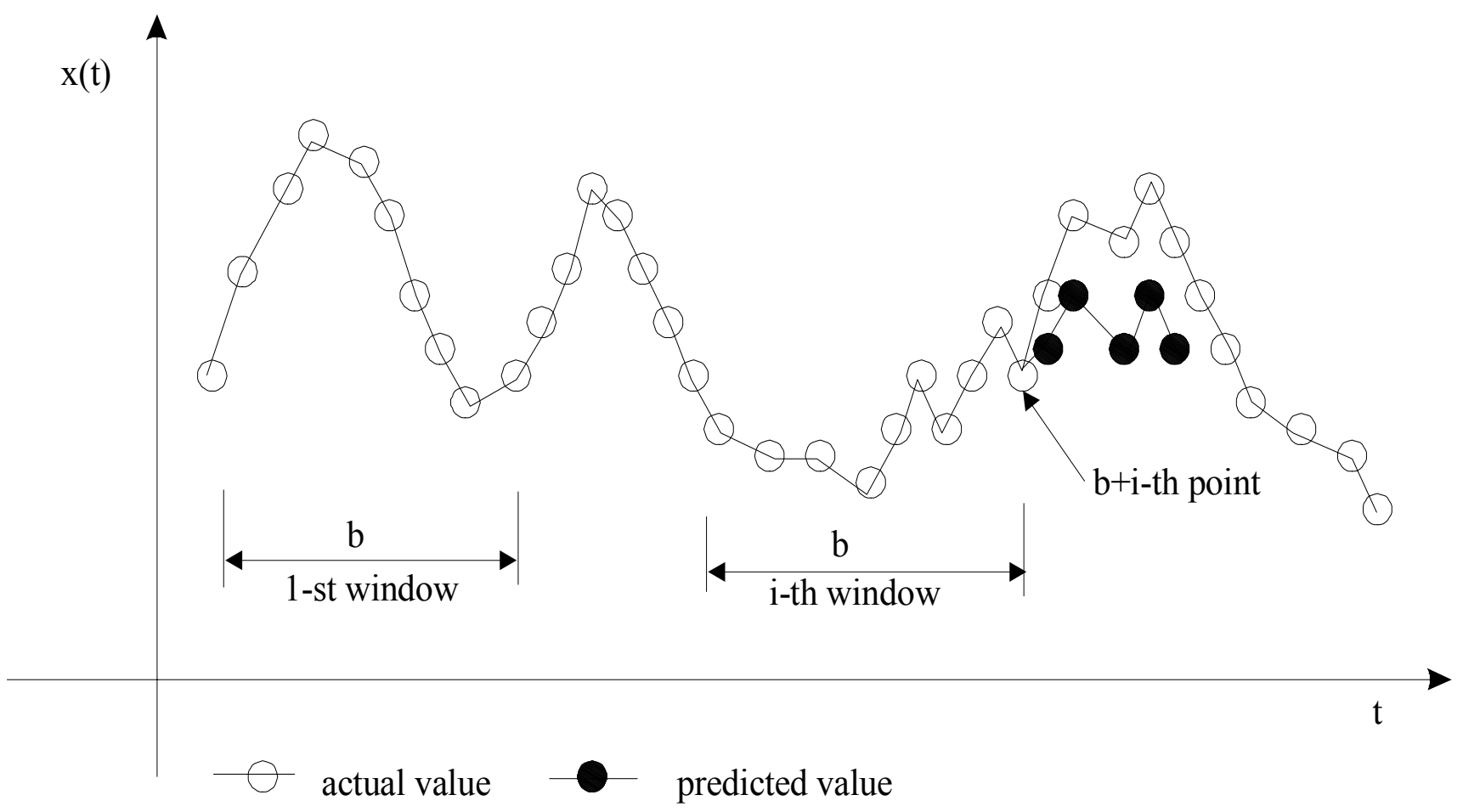

Fig. 1 - Method of moving window for identification of sharp changes.

is to investigate possibility of such detection by tracking of first local Lyapunov exponent.

The following algorithm used for the estimation of local Lyapunov exponent[11].
1.) Reconstructed vectors (5) created for timeseries of length $b$ in accordance with abovementioned procedures for finding of $\tau$ and D. 
2.) Multi-layer network with $D$ input neurons, sufficient number of hidden neurons and single output trained for prediction of next value of series for every piece.

3.) At $k$ - th point small perturbation $\left(\approx 10^{-8}\right)$ created.

4.) Perturbed and unperturbed series simulated by one-step prediction $\Delta t$ times and Euclidean distance between both of them $d(i), i=1, \ldots, \Delta t$ estimated.

5.) Plot $\log (d(i))$ vs. $i$ created and slope of leastsquare fitted line for it would be estimator of $\lambda_{k}^{\Delta t}$.

Two approaches for our investigation would be used. First, we would create network and train it for the whole time-series (refer it WS - whole series). Second, we would create separate network for each window (refer it MW - moving window).

\section{RESULTS AND DISCUSSIONS.}

Author investigate three time-series from US industrial volumes of shipment (www.economagic.com):

1.) aircraft industry;

2.) information technologies;

3.) motor vehicles (Fig. 2).

Multi-layer neural network was trained in accordance to above-mentioned algorithm to estimate $E(i) 100$ times and mean value of prediction error was estimated (Fig. 3).

Then, multi-layer network was trained to estimate local Lyapunov exponents in accordance to abovementioned algorithm 100 times. Mean value in each case was about 0.043 and fluctuate with time very weak. But standard deviation changed noticeably (Fig. 4).

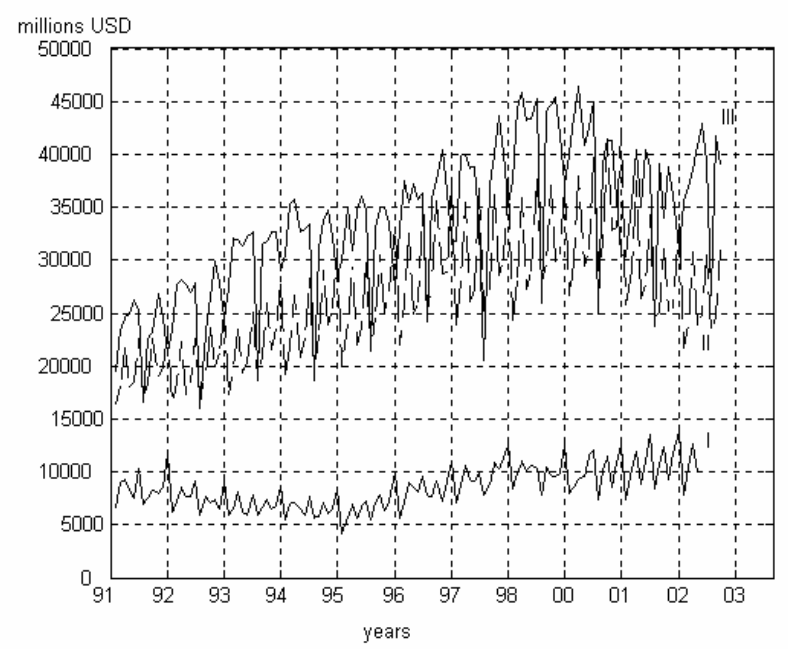

Fig. 2 - Original time-series (I - aircraft industry; II - information technologies; III - motor vehicles).

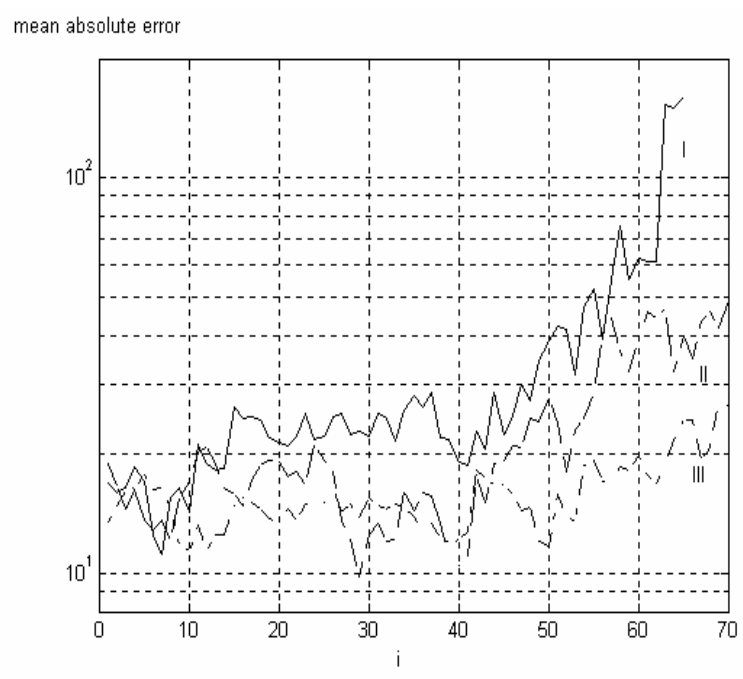

Fig. 3 - Mean absolute error for time-series.

For estimation relation between these values, we would use cross-correlation function, which is:

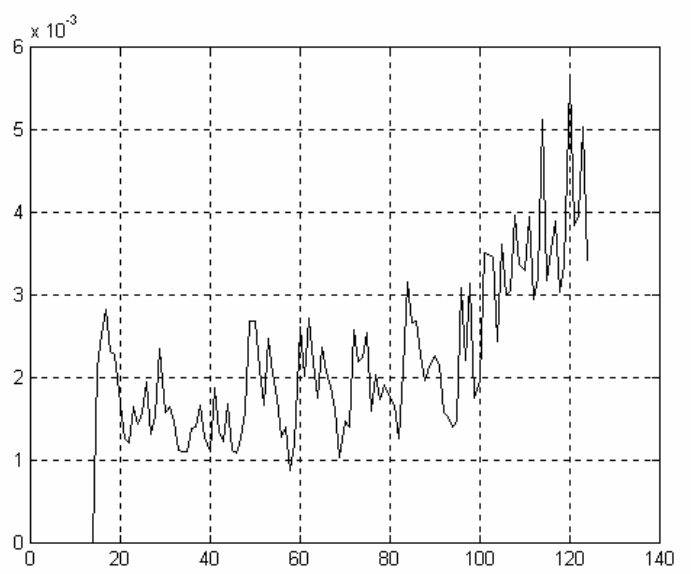

Fig. 4 - Fluctuation of standard deviation upon 100 realizations of LLE for first series.

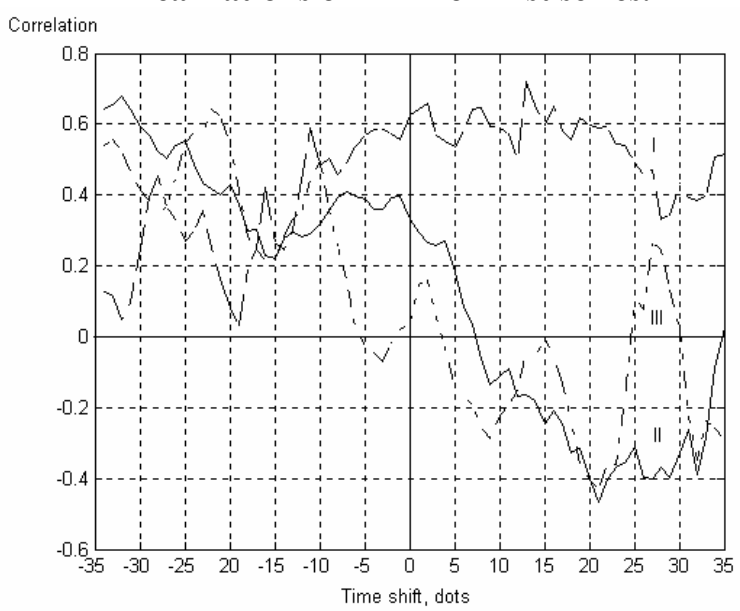

Fig. 5 - Cross-correlation functions between standard deviation of LLE and mean value of prediction error.

$$
C(x, y, \Delta t)=\frac{\operatorname{cov}(x(t), y(t+\Delta t))}{\sigma(x) \sigma(y)}
$$


where $\sigma(\cdot)$-standard deviation, $\operatorname{cov}(\cdot, \cdot)$ covariation between two realizations, which is

$$
\operatorname{cov}(x, y)=\frac{1}{n} \sum_{i=1}^{n} x(i) y(i)-\bar{x} \cdot \bar{y}
$$

where $\bar{x}, \bar{y}$ - mean values.

Correlation between standard deviation of local Lyapunov exponents and mean prediction error with shift from -35 to +35 was estimated (Fig 5).

As we can see, largest values of correlation occur in left half of plot, i.e. changes in local Lyapunov exponents anticipate changes in observations.

Analogously, cross-correlation functions were estimated for moving window approach. Neural network for each window trained and local Lyapunov exponents was estimated. In a first case, we estimate mean value for each window (Fig. 6). Then, mean Fourier frequency was estimated (Fig. 7).

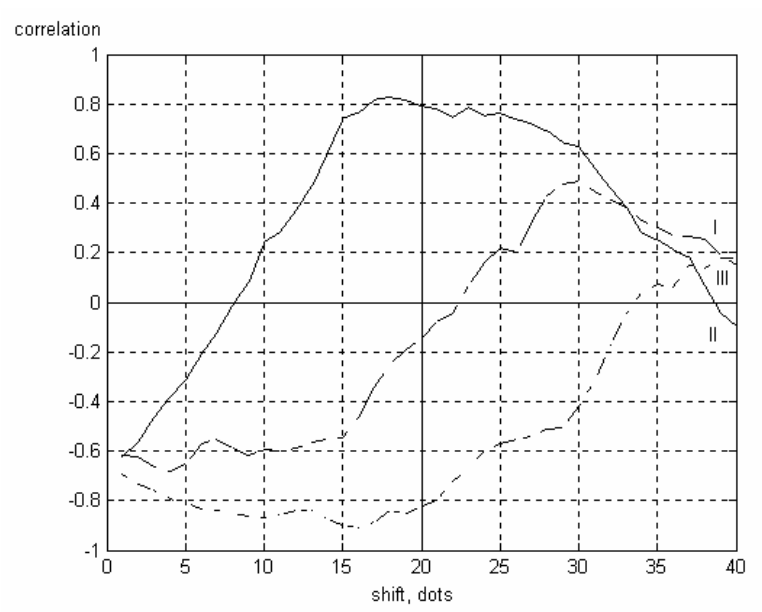

Fig.6 - Cross-correlation functions between mean value of LLE (moving window) and mean prediction error.

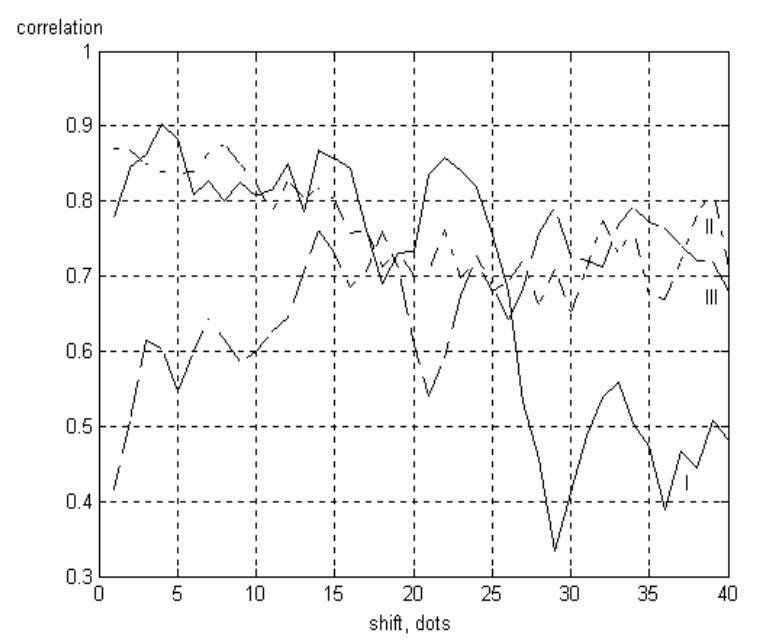

Fig. 7 - Cross-correlation functions between mean Fourier frequency of LLE (moving window) and mean prediction error.
As we can see, in first case sufficient value occurs for prediction 20-30 dots before, i. e. 1.5-2.5 years. It is out of reality for economical systems, and rather can be explained by another version than lag between systems changes and changes in observations.

Conversely, in case of mean Fourier frequency, we have sufficient large (0.8-0.9) mean of correlation for short-time prediction.

Thus, estimation of mean Fourier frequency of local Lyapunov exponents can help us in prediction and early detection of sharp changes.

There is some prospective of further research in this area. At first, early detection of changes in economics gives us insight in grounds of these changes. Then, exact detection of points of changes let us to establish cause effect relations between different indicators. And finally, notion of stability in relation to sharp changes can be established.

We would refer as stability in relation to sharp changes the property of system to keep its characteristics after sharp changes in environment. If we would have appropriate measures for estimation of intensity of this changes, then we could estimate rate of stability.

\section{CONCLUSIONS}

An approach to detect sharp changes was developed in this paper. At first, some specialties of prediction of economical time-series were pointed. Then, some introduction in method of nonlinear dynamics was given, with explanation of local Lyapunov exponents, Takens' theorem and describing of algorithms, which we need for further researches. Then, author's approach was developed and some numerical results were given.

\section{REFERENCES}

[1] H. Schuster. Deterministic chaos. Introduction. Moscow, 1988.

[2] P. Chen. A Random-Walk or Color-Chaos on the Stock Market? Time-Frequency Analysis of S\&P Indexes, Studies in Nonlinear Dynamics \& Econometrics, 1(2) (1996). p. 87-103.

[3] P. Chen. Empirical and Theoretical Evidence of Monetary Chaos. System Dynamics Review, 4 (1988), p. 81-108.

[4] V. I. Oseledec. Multiplicative Ergodic Theorem and Lyapunov Exponents for Dynamical Systems. Trans. of Mosc. Math. Soc., 19 (1968), p. 179-210.

[5] J.-P. Eckmann, D. Ruelle. Ergodic Theory of Chaos and Strange Attractors. Rev. Mod. Phys., 57 (1989), p. 617-656.

[6] H. D. I. Abarbanel, R. Brown, M. B. Kennel. Lyapunov Exponents in Chaotic Systems: Their 
Importance and Their Evaluation Using Observed Data. Int. J. Mod. Phys., B5 (1991) p. 1347-1375.

[7] F. Takens. Detecting Strange Attractors in Turbulence. Dynamical Systems and Turbulence. Lecture Notes in Mathematics. Ed. by D. A. Rand, L. S. Young. Heidelberg, 1981, p. 366-381.

[8] A. M. Fraser, H. L. Swinney. Independent Coordinates for Strange Attractors from Mutual Information. Phys. Rev. A 33, 1986, p. 1134-1140.

[9] M. B. Kennel, R. Brown, H. D. I. Abarbanel. Determining Embedding Dimension for PhaseSpace Reconstruction Using a Geometrical Construction. Phys. Rev. A 45, 1987, p. 3403-3411.

[10] G. Benettin, L. Galgani, J. M. Strelcyn. Kolmogorov Entropy and Numerical Experiments. Phys. Rev. 14, 1976, p. 2338-2345.

[11] V. Golovko, Y. Savitsky, N. Maniakov. Neural Networks for Signal Processing in Measurement Analysis and Industrial Applications: the Case of Chaotic Signal Processing. Chapter in NATO book "Neural Networks for Instrumentation, Measurement and Related Industrial Application". Amsterdam: IOS Press, 2003, p119-143.

[12]B. A. Bailey, S. Ellner, D. W. Nychka. Chaos with Confidence: Asimptotics and Bifurcations of Local Lyapunov Exponents. Proceeding of the fields. CRM workshop on Nonlinear Dynamics and Time-Series: Building a Bridge between the Natural and Statistical Science 11, 1997, p. 115-133.

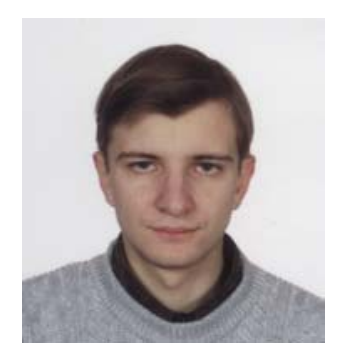

Dmitry Maliuk. Date of birth 1977, October, 4. In 1999 graduate from Brest Polytechnical Institute. In 2004 graduate from postgraduate studies in Brest State Technical University with Ph. D. work "Robustness of civil engineering firms".

Current position: assistant of "Civil engineering economics and arrangement" department of Brest State Technical University.

Scientific intersets: robustness of economic entities, artificial intellegence, neural and agentbased modelling in economics, real estate appraisal.

\section{Georgy Boyarintsev.}

Date of birth 1951, January, 27. In 1973 graduate from Brest Building-engineering Institute on speciality "Civil Engineering". In 1984 take Ph. $D$. in economics. Dissertation title "Development of control system in farm engineering entities on example of "Mezhkolkhozstroy"corporation".

Current position: professor of "Civil engineering economics and arrangement" department of Brest State Technical University.

Scientific interests: arrangement and management in civil engineering firms, differentiation in construction sector, applied mathematics in economics. 